\title{
Role of Postoperative Radiotherapy in Nonmetastatic Head and Neck Adenoid Cystic Carcinoma
}

Yue Chen, MD ${ }^{1,2,3,4, *}$; Zi-Oi Zheng, MD ${ }^{1,2,3,4, *}$; Fo-Ping Chen, MD1,2,3,4,*; Jian-Ye Yan, BA ${ }^{5, *} ;$ Xiao-Dan Huang, MD ${ }^{1,2,3,4}$; Feng Li, MD 1,2,3,4; Ying Sun, MD 1,2,3,4; and Guan-Qun Zhou, MD 1,2,3,4

\section{ABSTRACT}

Background: Head and neck adenoid cystic carcinoma (ACC) is a rare malignant tumor that is prone to local recurrence. The NCCN Guidelines for Head and Neck Cancers recommend that all patients with ACC receive postoperative radiotherapy (PORT). However, whether PORT can improve local control and which patients can benefit from PORT are unknown. This study aimed to assess the role of PORT and provide individualized suggestions for postoperative therapy in patients with ACC. Patients and Methods: We retrospectively reviewed patients with nonmetastatic head and neck ACC who underwent surgery with or without PORT. Recursive partitioning analysis (RPA) was performed to categorize the patients and predict local recurrence-free survival (LRFS). The survival outcome was compared between non-PORT and PORT groups. Results: A total of 319 patients were included. PORT was identified as a prognostic factor for LRFS in univariate $(P=.01)$ and multivariate analysis $(P<.01)$. However, it did not improve distant metastasis-free survival, diseasefree survival, or overall survival in univariate analysis. RPA categorized patients into 3 prognostic groups: low-risk (negative margin, T1-T2, primary location $=$ major or minor salivary gland), intermediate-risk (negative margin, T1-T2, primary location = other locations instead of a major or minor salivary gland; negative margin, T3-T4; positive margin, without bone invasion), and high-risk (positive margin, with bone invasion). Significant LRFS improvements in the PORT group were observed among intermediate-risk $(P<.01)$ and high-risk patients $(P<.05)$. LRFS improvements among low-risk patients were relatively insignificant $(P=.10)$. Conclusions: PORT was shown to be a positive prognostic factor for improved LRFS in ACC. Furthermore, PORT could significantly improve LRFS in intermediate-risk and highrisk patients with $A C C$, but whether low-risk patients could benefit from PORT needs further study.

J Natl Compr Canc Netw 2020;18(11):1476-1484 doi: $10.6004 /$ jnccn.2020.7593

\footnotetext{
${ }^{1}$ Department of Radiation Oncology, Sun Yat-sen University Cancer Center, ${ }^{2}$ State Key Laboratory of Oncology in South China, ${ }^{3}$ Collaborative Innovation Center of Cancer Medicine, and ${ }^{4}$ Guangdong Key Laboratory of Nasopharyngeal Carcinoma Diagnosis and Therapy, Guangzhou, People's Republic of China; and ${ }^{5}$ Department of Radiation Oncology, Wuzhou Red Cross Hospital, Wuzhou, Guangxi, People's Republic of China.
}

*These authors contributed equally.

\section{Background}

Adenoid cystic carcinoma (ACC) is a rare low-grade malignant tumor, accounting for approximately $1 \%$ of all head and neck malignancies. ${ }^{1}$ ACC usually originates from many types of glands and most often arises in salivary glands, accounting for $25 \%$ of malignant tumors in the major salivary glands and $50 \%$ in the minor glands. ${ }^{2}$ The natural disease process is characterized by prolonged progression and multiple recurrences. ${ }^{3,4}$

Surgery has been considered the most important treatment of ACC. ${ }^{5}$ However, because of the unique anatomic structures of the head and neck, the scope of surgery is often limited. Moreover, ACC is prone to local nerve invasion, and therefore it is often difficult to remove the tumor completely. Consequently, postoperative radiotherapy (PORT) is often considered an important adjuvant treatment after surgery. The NCCN Clinical Practice Guidelines in Oncology (NCCN Guidelines) for Head and Neck Cancers have a category $2 \mathrm{~B}$ recommendation for patients with T1 ACC and a category $2 \mathrm{~A}$ recommendation for other patients with ACC to receive additional PORT after complete resection. ${ }^{6}$ However, whether PORT can improve local control in patients with ACC remains controversial, ${ }^{3,5,7-9}$ and which patients need additional PORT often confuses surgeons and radiologists.

Given the rarity of ACC and the difficulty of conducting relevant prospective clinical trials, we conducted a retrospective study of a large sample of patients with nonmetastatic head and neck ACC to elucidate the role of PORT in survival outcome, which would help surgeons and radiologists provide individualized recommendations regarding PORT to patients with ACC.

\section{Patients and Methods}

Patient Characteristics

From January 2000 through December 2017, 480 patients were pathologically diagnosed with head and neck ACC and treated in Sun Yat-sen University Cancer Center in

See JNCCN.org for supplemental online content. 
China. The following patients were excluded: 115 patients who received their initial surgery in other hospitals without accurate baseline data, 31 who did not receive surgery, 14 with initial distant metastasis, and 9 lost to follow-up within 3 months after treatment. Therefore, we focused on 319 patients who had undergone surgery with or without PORT after the first diagnosis. The characteristics of the 319 patients are shown in Table 1. Detailed primary sublocations of ACC are shown in supplemental eTable 1 (available with this article at JNCCN.org). In our study, 32 patients with nerve invasion did not receive PORT: 10 could not afford the cost of PORT; 8 due to elderly age; 7 failed to follow doctors' orders; 6 due to wound infection, poor healing, and weakness; and 1 worried about the adverse effects of radiotherapy (RT) and chose postoperative chemotherapy.

\section{Treatment Methods}

\section{Surgery}

Of the 319 patients included, 92 (28.8\%) underwent surgery alone and the remaining $227(71.2 \%)$ received PORT. As for the operative method, $232(72.7 \%)$ underwent resection of the primary location alone, whereas $87(27.3 \%)$ also received resection of cervical lymph nodes. Indications for neck dissection were as follows: clinically positive lymph nodes; large T tumors; patients whose neck would be perforated or open during operation; or patients who needed free cervical flaps for repair. The extent of lymph node dissection was at the surgeon's discretion, mainly based on the patients' medical histories, clinical palpation, imaging data, and intraoperative exploration. If there were several clinically positive lymph nodes, a large volume of lymph nodes, extranodal invasion, or T3-T4 tumors, unilateral cervical lymph node resection was performed. Otherwise, limited regional lymph node dissection was performed. Of the 87 patients who underwent neck dissection, 6 nodes per patient were dissected on average.

\section{Radiotherapy}

Indications for PORT included large primary tumor, nerve invasion, positive margins, or the surgeons considered the primary tumor unresectable and recommended PORT. Different RT techniques were used: 81 patients received 2D RT, 25 received 3D conformal RT (3D-CRT), and 213 received intensity-modulated RT (IMRT). For patients treated with 3D-CRT and IMRT, the gross tumor volume (GTV) of the RT target was delineated basing on preoperative and postoperative contrast-enhanced CT or contrast-enhanced MRI, including the region with positive margin and persistent enhancement after surgery. In patients with large $\mathrm{T}$ tumor invading deep tissues or the skull base that could

\begin{tabular}{|c|c|c|c|c|}
\hline Characteristic & $\begin{array}{l}\text { Total } \\
\text { n (\%) }\end{array}$ & $\begin{array}{c}\text { Non-PORT } \\
\text { n (\%) }\end{array}$ & $\begin{array}{l}\text { PORT } \\
\text { n (\%) }\end{array}$ & $\begin{array}{c}P \\
\text { Value }\end{array}$ \\
\hline Total, N & 319 & 92 & 227 & \\
\hline Age, $y$ & & & & .03 \\
\hline$\leq 47$ & $169(53.0)$ & $40(43.5)$ & $129(56.8)$ & \\
\hline$>47$ & $150(47.0)$ & $52(56.5)$ & $98(43.2)$ & \\
\hline Sex & & & & .44 \\
\hline Male & $139(43.6)$ & $37(40.2)$ & $102(44.9)$ & \\
\hline Female & $180(56.4)$ & $55(59.7)$ & $125(55.0)$ & \\
\hline Alcohol history & & & & .64 \\
\hline No & $306(95.9)$ & $89(96.7)$ & $217(95.6)$ & \\
\hline Yes & $13(4.1)$ & $3(3.3)$ & $10(4.4)$ & \\
\hline Tobacco history & & & & .74 \\
\hline No & $267(83.7)$ & $76(82.6)$ & $191(84.1)$ & \\
\hline Yes & $52(16.3)$ & $16(17.4)$ & $36(15.9)$ & \\
\hline Primary tumor location & & & & .14 \\
\hline Major salivary gland & $97(30.4)$ & $35(38.0)$ & $62(27.3)$ & \\
\hline Minor salivary gland & $189(59.2)$ & $47(51.1)$ & $142(62.6)$ & \\
\hline Other & $33(10.3)$ & $10(10.9)$ & $23(10.1)$ & \\
\hline Nerve invasion & & & & .82 \\
\hline No & $211(66.1)$ & $60(65.2)$ & $151(66.5)$ & \\
\hline Yes & 108 (33.9) & $32(34.8)$ & $6(2.6)$ & \\
\hline Vessel invasion & & & & .52 \\
\hline No & $301(94.4)$ & $88(95.7)$ & $213(93.8)$ & \\
\hline Yes & $18(5.6)$ & $4(4.3)$ & $14(6.2)$ & \\
\hline Bone invasion & & & & $<.01$ \\
\hline No & $242(75.8)$ & $81(88.0)$ & $161(70.9)$ & \\
\hline Yes & $77(24.1)$ & $11(12.0)$ & $66(29.1)$ & \\
\hline Surgical margin & & & & .01 \\
\hline Negative & $239(74.9)$ & $78(84.8)$ & $161(70.9)$ & \\
\hline Positive & $80(25.1)$ & $14(15.2)$ & $66(29.1)$ & \\
\hline T stage & & & & $<.01$ \\
\hline $\mathrm{T} 1-\mathrm{T} 2$ & $146(45.8)$ & $55(60.0)$ & $91(40.1)$ & \\
\hline T3-T4 & $173(54.2)$ & $37(40.0)$ & $136(59.9)$ & \\
\hline Lymph node metastasis & & & & .38 \\
\hline No & $302(94.7)$ & $85(92.4)$ & 217 (95.6) & \\
\hline Yes & $17(5.3)$ & $7(7.6)$ & $10(4.4)$ & \\
\hline Concurrent chemotherapy & & & & - \\
\hline No & - & - & $200(88.1)$ & \\
\hline Yes & - & - & $27(11.9)$ & \\
\hline
\end{tabular}

Abbreviation: PORT, postoperative radiotherapy.

hardly be completely removed by surgery, GTV included these sites. The clinical target volume (CTV) extended 5 to $10 \mathrm{~mm}$ beyond the GTV. If the tumor invaded the main nerves, the CTV included not only the postoperative primary bed but also the potential path of the 
main nerves along with intracranial or peripheral invasion, examples of which are shown in supplemental eFigures 1 and 2. As for pathologically positive cervical lymph nodes, a certain cervical region was included in the CTV. The planning target volume (PTV) was a 5-mm extension of CTV. In $92 \%$ of cases, the planning target volume of the GTV and/or the involved lymph nodes was generally given as a total dose of 60 to $72 \mathrm{~Gy}$. In patients with residual primary tumors or positive lymph nodes, the prescription dose was 66 to 72 Gy. For patients with adverse features but the tumor was completely resected, the dose was 60 to $66 \mathrm{~Gy}$.

\section{Patient Follow-Up and Statistical Analysis}

After the first visit to our hospital, the patients were followed up every 3 months in the first 2 years, and every 6 months thereafter until the patients died. Local recurrence-free survival (LRFS) was defined as the time from the beginning of treatment to the first recurrence at the primary location. ${ }^{7}$ Patients with regional recurrence were not included. Distant metastasis-free survival (DMFS) was defined as the time from the beginning of

A

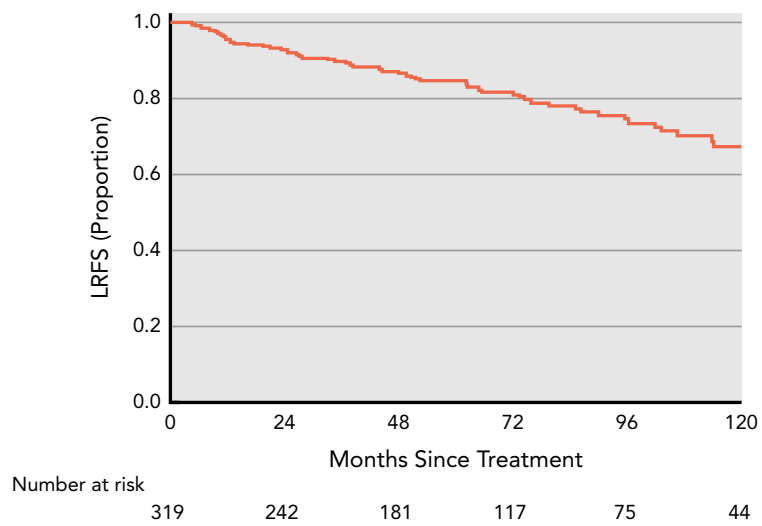

C

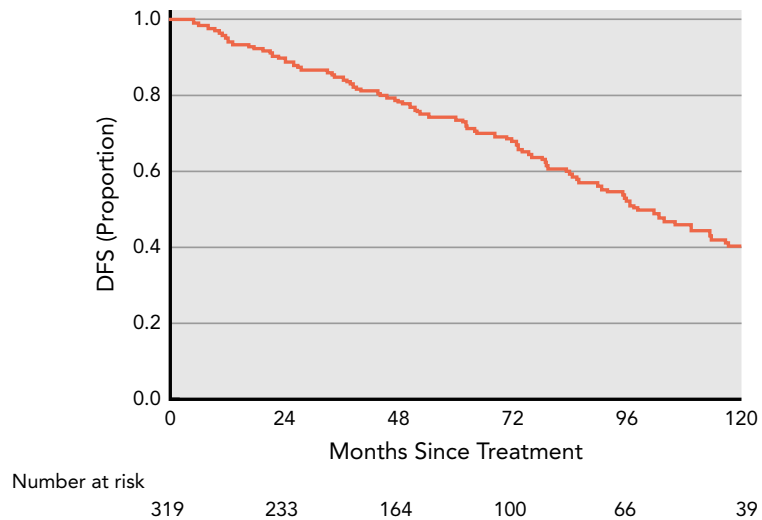

treatment to the first recurrence at a distant location. Pathologic confirmation or radiographic evidence (CT, MRI, or PET/CT) was necessary for the diagnosis of local recurrence or distant metastasis. Overall survival (OS) was defined as the time from the beginning of treatment to death or the last follow-up. Disease-free survival (DFS) was defined as the time from the beginning of treatment to tumor progression, death, or the last follow-up.

Patients were divided into 2 groups based on whether they received PORT or not: PORT and nonPORT. The clinicopathologic factors of these groups were compared using the chi-square test. Cumulative survival rates were estimated using the Kaplan-Meier method and compared with the log-rank tests. Multivariate Cox regression analysis was performed to confirm the role of PORT in LRFS when adjusted for other possible factors by using the enter algorithm. We included age, sex, alcohol history, tobacco history, primary tumor location, nerve invasion, vessel invasion, bone invasion, surgical margin, $\mathrm{T}$ stage, lymph node metastasis, and PORT in the Cox model. Moreover, forest plots were generated to summarize the adjusted hazard ratios (HRs)

B

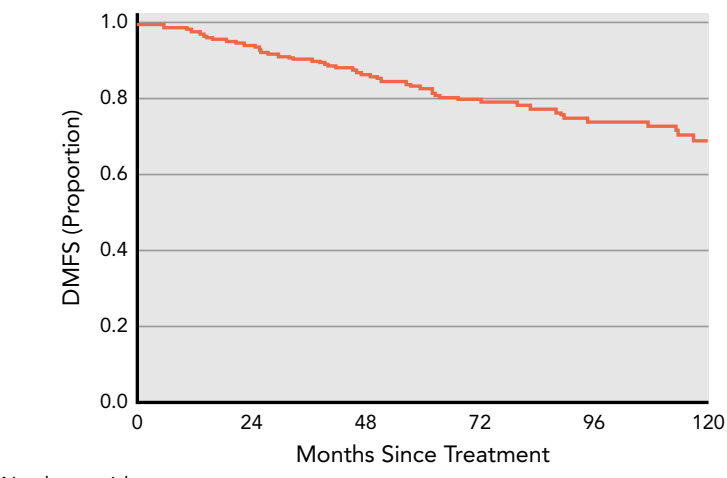
7

D

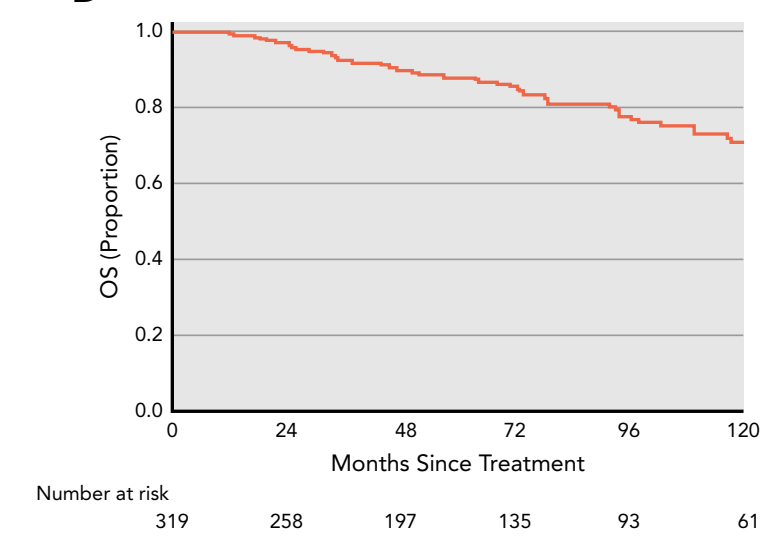

Number at risk

Figure 1. Rates of (A) LRFS, (B) DMFS, (C) DFS, and (D) OS in patients with adenoid cystic carcinoma.

Abbreviations: DFS, disease-free survival; DMFS, distant metastasis-free survival; LRFS, local recurrence-free survival; OS, overall survival. 
with $95 \%$ confidence intervals for the validated predictors of LRFS.

Recursive partitioning analysis (RPA) for LRFS was based on the same variables except for PORT. To ensure a sufficient number of patients were included in the analysis of subgroups, a viable split was considered when at least 50 patients were in each group and the $P$ value was statistically significant, as described elsewhere. ${ }^{10}$ To attain the patients most likely to benefit from PORT, we performed individual comparisons of non-PORT and PORT for each risk group. Statistical analyses were performed using SPSS Statistics, version 24 (IBM Corp) or the RMS package in R version 3.5.2 (R Foundation for Statistical Computing). The forest plots were generated using Microsoft Excel (Microsoft Inc). All $P$ values were 2 -sided with significance defined as a $P<.05$.

\section{Results}

\section{Survival Outcomes}

After a median follow-up of 64 months (range, 5-227 months), 63 of 319 patients (20\%) developed local recurrence, 64 (20\%) developed distant metastasis, 133 (42\%) experienced tumor progression or death, and 64 (20\%) died. In our study, 29 people died within 5 years (25 died of a tumor, 2 of natural aging, 1 of cardiovascular and cerebrovascular diseases, and 1 because of an accident); 22 people died between 5 and 10 years (16 died of a tumor, 3 of cardiovascular and cerebrovascular diseases, 2 of natural aging, and 2 because of an accident).

Survival curves are shown in Figure 1. For the whole cohort, the 1-, 3-, 5-, and 10-year LRFS rates were 98\%, $90 \%, 85 \%$, and $66 \%$, respectively; DMFS rates were $98 \%, 91 \%, 83 \%$, and $69 \%$, respectively; DFS rates were $98 \%, 80 \%, 67 \%$, and $40 \%$, respectively, and OS rates were $100 \%, 92 \%, 88 \%$, and $71.1 \%$, respectively.

\section{Effect of PORT on LRFS}

Patients in the PORT group were younger, with a greater proportion of bone invasion, positive surgical margins, and advanced $\mathrm{T}$ stage compared with the non-PORT group (Table 1). The 5-year LRFS, DMFS, DFS, and OS were $75 \%, 94 \%, 66 \%$, and $90 \%$, respectively, for the nonPORT group and $89 \%, 79 \%, 67 \%$, and $88 \%$, respectively, for the PORT group (Table 2).

In univariate analysis, PORT was a significant prognostic factor for LRFS (HR, 0.54; 95\% CI, 0.33-0.89; $P=.01$ ) but not for DMFS (HR, 3.38; 95\% CI, 1.65-6.95; $P<.01$ ), DFS (HR, 1.10; 95\% CI, 0.76-1.60; $P=.61$ ), or OS (HR, 1.32; 95\% CI, 0.76-2.30; $P=.32$ ) (Table 2). After adjusting for covariates, PORT remained an independent prognostic factor for LRFS (HR, 0.27; 95\% CI, 0.15-0.48; $P<.01$ ) in multivariate analysis (Figure 2).

\begin{tabular}{|c|c|c|c|c|}
\hline Outcome & $\begin{array}{c}\text { Non-PORT } \\
(n=92)\end{array}$ & $\begin{array}{c}\text { PORT } \\
(n=227)\end{array}$ & $\begin{array}{c}\text { HR } \\
(95 \% \mathrm{Cl})\end{array}$ & $\begin{array}{c}P \\
\text { Value }^{a}\end{array}$ \\
\hline LRFS rate & & & $0.54(0.33-0.89)$ & .01 \\
\hline 1-year & $92 \%$ & $98 \%$ & & \\
\hline 3-year & $86 \%$ & $92 \%$ & & \\
\hline 5 -year & $75 \%$ & $89 \%$ & & \\
\hline 10-year & $61 \%$ & $68 \%$ & & \\
\hline DMFS rate & & & $3.38(1.65-6.95)$ & $<.01$ \\
\hline 1 -year & $100 \%$ & $97 \%$ & & \\
\hline 3-year & $96 \%$ & $89 \%$ & & \\
\hline 5 -year & $94 \%$ & $79 \%$ & & \\
\hline 10-year & $84 \%$ & $63 \%$ & & \\
\hline DFS rate & & & $1.10(0.76-1.60)$ & .61 \\
\hline 1-year & $92 \%$ & $97 \%$ & & \\
\hline 3-year & $82 \%$ & $80 \%$ & & \\
\hline 5-year & $66 \%$ & $67 \%$ & & \\
\hline 10-year & $45 \%$ & $37 \%$ & & \\
\hline OS rate & & & $1.32(0.76-2.30)$ & .32 \\
\hline 1-year & $99 \%$ & $99 \%$ & & \\
\hline 3-year & $93 \%$ & $92 \%$ & & \\
\hline 5 -year & $90 \%$ & $88 \%$ & & \\
\hline 10-year & $71 \%$ & $71 \%$ & & \\
\hline
\end{tabular}

Abbreviations: DFS, disease-free survival; DMFS, distant metastasis-free survival; HR, hazard ratio; LRFS, local recurrence-free survival; OS, overall survival; PORT, postoperative radiotherapy.

a $P$ values were calculated by log-rank test.

\section{RPA Model for LRFS}

The RPA model was generated based on the endpoint of LRFS. The RPA algorithm segregated the 319 patients with ACC into 5 classes (Figure 3). Because the LRFS of classes 2, 3 , and 4 were not significantly different (class 2 vs $3, P=.53$; class 2 vs $4, P=.89$; class 3 vs $4, P=.46$ ), these 3 groups were combined. Therefore, the final RPA model categorized patients into the following 3 prognostic groups: low-risk (T1-T2 disease with negative margin, and primary location limited to the major or minor salivary gland; $n=112$ ), intermediaterisk (T1-T2 disease with negative margin and the primary tumor located in other sites except salivary gland; T3-T4 disease with negative margin; or positive margin but without bone invasion; $n=167$ ), and high-risk (positive margin with bone invasion; $\mathrm{n}=40$ ), with corresponding 5 -year LRFS rates of $93 \%, 83 \%$, and $44 \%$, respectively (low-risk group: HR, 1.00; intermediate-risk group: HR, 3.12; 95\% CI, 1.55-6.29; $P<.01$; high-risk group: HR, 7.60; 95\% CI, 3.39-17.05; $P<.01$ ).

\section{Role of PORT in the Risk Groups}

The LRFS of the 3 risk groups categorized by RPA were significantly different from each other (all $P<.017$; Figure 4A). LRFS for each of the 3 groups is shown in 


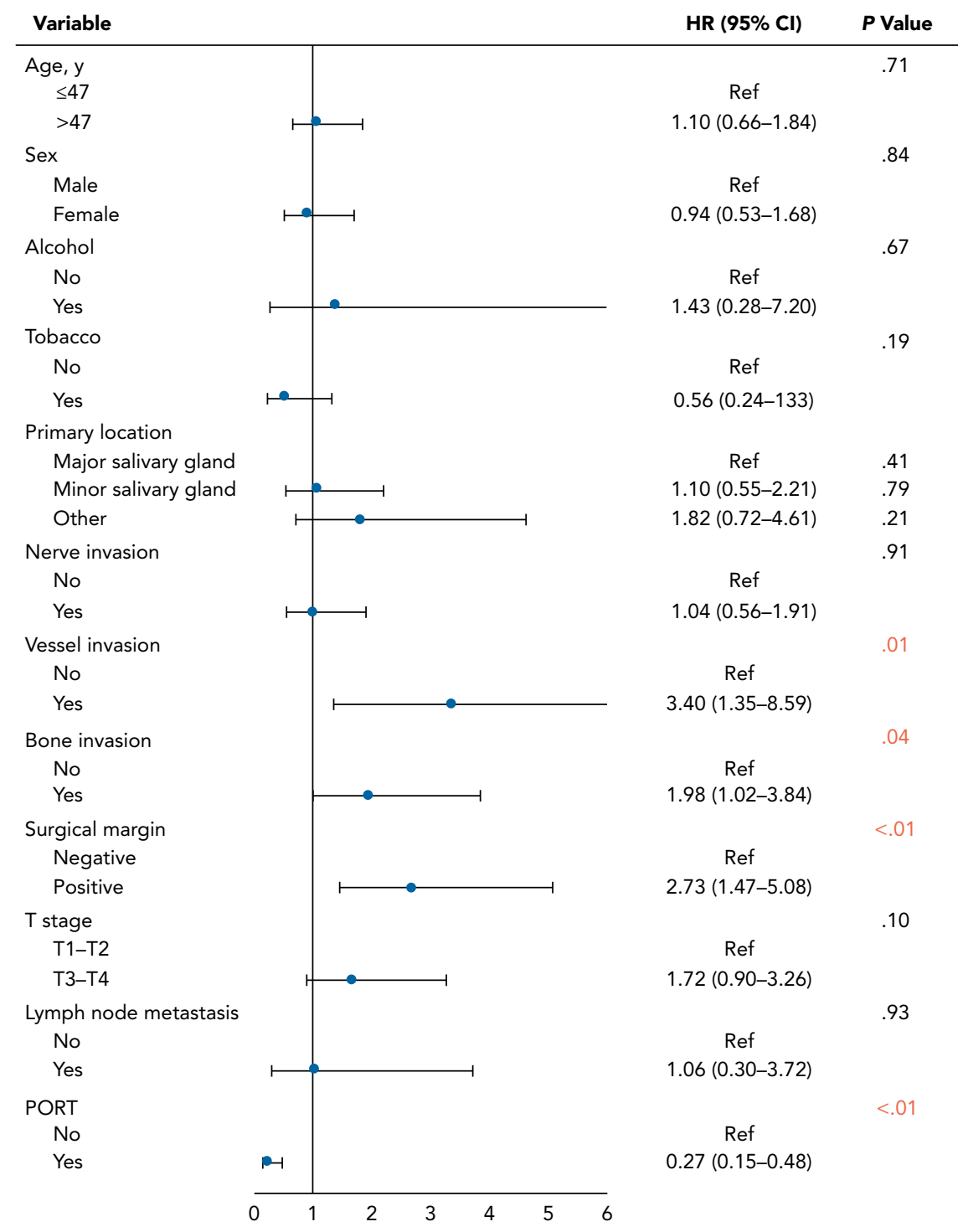

Figure 2. Forest plot analysis of local recurrence-free survival in patients with adenoid cystic carcinoma. Red $P$ values are statistically significant. Abbreviations: HR, hazard ratio; PORT, postoperative radiotherapy.

Figure $4 \mathrm{~B}, \mathrm{C}, \mathrm{D}$, respectively. Compared with the nonPORT group, the patients receiving PORT had better LRFS in the intermediate-risk ( $\mathrm{HR}, 0.39 ; 95 \% \mathrm{CI}, 0.20-0.74$; $P<.01$ ) and high-risk groups (HR, 0.34; 95\% CI, 0.11-1.05; $P<.05)$, whereas the difference was not significant in the low-risk group (HR, 0.34; 95\% CI, 0.09-1.30; $P=.10$ ).

Other than LRFS, we also studied the role of PORT in DMFS. The $P$ values for DMFS for the 3 risk groups were $<0.01,0.23$, and 0.28 , respectively, and the patients with ACC failed to benefit from PORT in all 3 risk groups (supplemental eFigure 3).

\section{Discussion}

Although PORT has been used widely in clinical practice, it was unclear whether it could reduce the local recurrence of ACC in patients, and which patients could benefit the most from this treatment. Our analysis showed that PORT can improve LRFS in patients with ACC; however, the benefit was more obvious in intermediate- and high-risk patients. Whether low-risk patients could benefit from PORT requires further study.

\section{Local Failure in Patients With ACC}

In our study, we found that the rate of LRFS, DMFS, DFS, and OS in patients with ACC had been decreasing continuously for 10 years after initial treatment. In contrast, tumor progression usually occurs within 5 years in other tumors, with the risk of recurrence or metastasis rates decreasing rapidly after 5 years. ${ }^{11-13}$ That is because ACC is a low-grade malignancy with slow growth. 


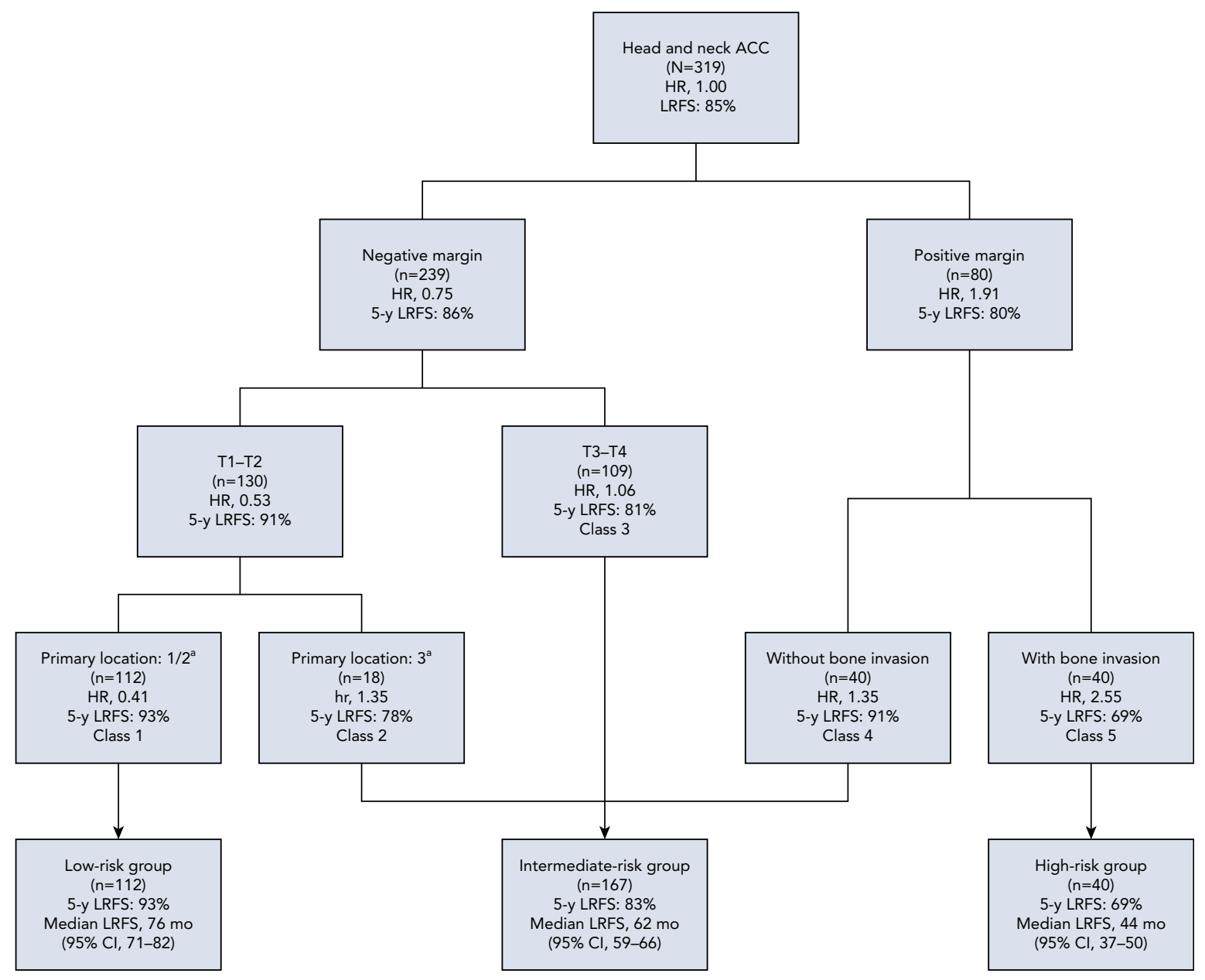

Figure 3. Prognostic grouping by recursive partitioning analysis in patients with head and neck ACC for predicting LRFS. Abbreviations: ACC, adenoid cystic carcinoma; HR, hazard ratio; LRFS, local recurrence-free survival.

aPrimary tumor location limited to the major or minor salivary gland.

bPrimary tumor located in sites other than the salivary glands.

Consistent with our findings, Jones et $\mathrm{al}^{14}$ found that the actuarial local recurrence rate was $83 \%$ at 20 years and increased to $100 \%$ at 30 years $(n=104)$. The 5 - and 10 year local control rates in a study by Chen et al ${ }^{15}$ were $88 \%$ and $77 \%$, respectively $(\mathrm{n}=140)$. Iseli et $\mathrm{al}^{5}$ reported that the LRFS rate was $68.2 \%$ at 5 years and $40.8 \%$ at 10 years $(n=183)$. We also found that the OS rate of ACC remained as high as $88 \%$ at 5 years and decreased rapidly to $71 \%$ at 10 years. A recent study by Ouyang et $\mathrm{al}^{16}$ that included $>210$ patients with ACC treated over 25 years reported 5- and 10 -year OS rates of $85 \%$ and $71 \%$, respectively, which were consistent with our data. These data suggest that ACC was characterized by an indolent but persistent course.

The mainstay treatment modality of ACC is surgery. Unfortunately, for ACC of the head and neck, the scope of surgery is largely limited by the complex and delicate anatomic structures. Typically, it is difficult to completely remove the tumor and protect the organs at risk simultaneously. Radical surgery can result in probable disfigurement of the patient. ${ }^{2,5,17}$ To preserve patients' quality of life after surgery, surgeons often tend to minimize the scope of surgery, which can lead to a decline in the local recurrence rate. ${ }^{18,19}$

Furthermore, our LRFS rate was $85 \%$ at 5 years and $66 \%$ at 10 years, which is similar to rates reported in previous studies. ${ }^{7,20}$ In our study, approximately $20 \%$ of patients eventually developed local recurrence, similar to the results of Zhang et $\mathrm{al}^{21}{ }^{21}$ whereas a study by Jang et $\mathrm{al}^{22}$ reported a local recurrence rate as high as $32 \%$. Because ACC has been widely reported as a highly recurrent tumor in many other research studies, ${ }^{5,7,18,23}$ local recurrence is one of the main patterns of treatment failure in patients with ACC. Consequently, the key to improving the efficacy of treatment in patients with ACC is to reduce local recurrence. ${ }^{24}$ 
A

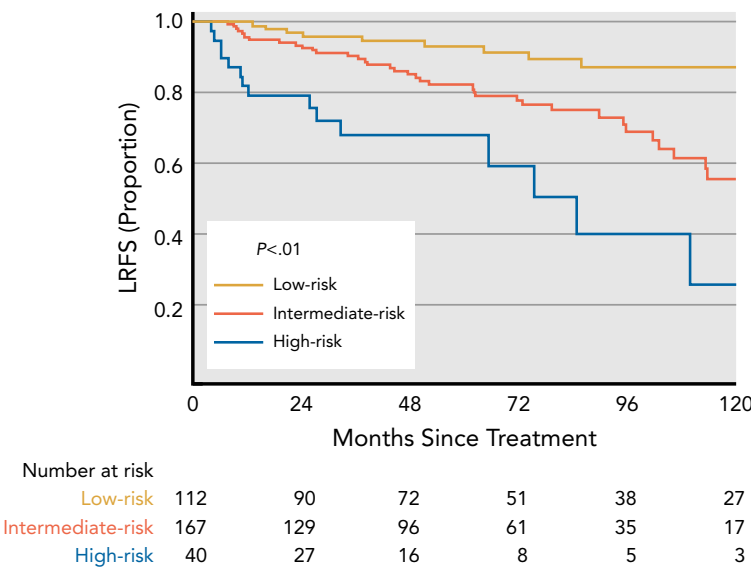

C

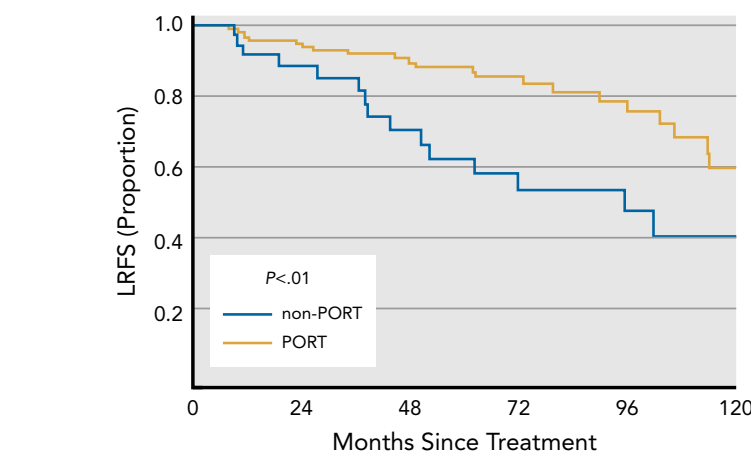

Number at risk non-PORT 38 PORT 129

$\begin{array}{rrrr}29 & 20 & 12 & 9 \\ 101 & 77 & 50 & 27\end{array}$

B

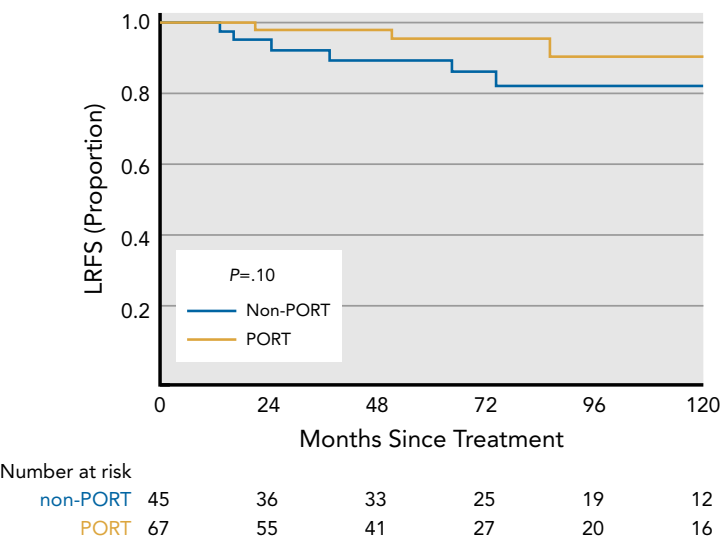

D

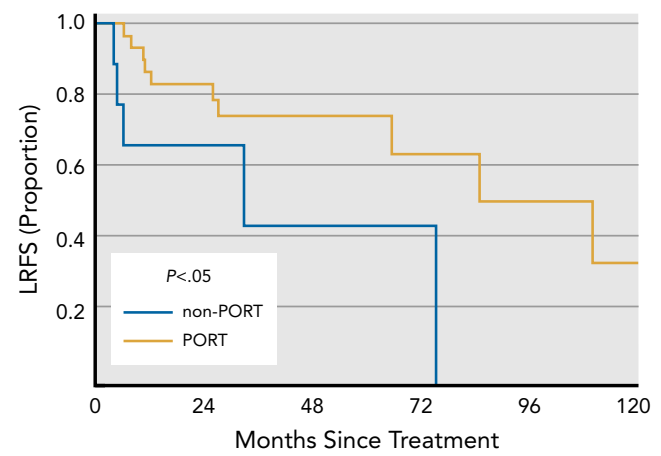

Number at risk

non-PORT 9

PORT 31

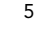

23

Figure 4. Kaplan-Meier estimates of LRFS in different prognostic groups derived by recursive partitioning analysis. (A) LRFS in 3 prognostic groups. (B) PORT in the low-risk group. (C) PORT in the intermediate-risk group. (D) PORT in the high-risk group.

Abbreviations: LRFS, local recurrence-free survival; PORT, postoperative radiotherapy.

\section{Benefits of PORT}

$\mathrm{RT}$ is one of the most important means of improving local control. It uses high-energy radiation to destroy the DNA of tumor cells via direct ionization or indirect ionization by photons or charged particles. ${ }^{25,26}$ The advantage of this technique over surgical treatment is that precise RT can protect normal tissues while killing tumors. A recent study of 87 patients with ACC over 20 years by Ali et $\mathrm{al}^{7}$ showed that patients who did not receive PORT had a 13fold increased risk of local failure compared with those treated with PORT after adjusting for stage. Therefore, PORT may serve as an important adjuvant treatment for high-risk patients (especially those with residual tumor). ${ }^{27,28}$

Our study showed that PORT could improve LRFS in patients with ACC but had no effect on DMFS, DFS, or OS, which is consistent with the findings of many other research studies. ${ }^{7,29,30}$ One reason for this is that ACC is a low-grade malignant tumor. If patients who experience local recurrence are treated promptly with salvage treatment, they can still attain a longer OS. Furthermore, patients are prone to distant metastasis, which is also one of the typical failure patterns of ACC. ${ }^{22,31}$ However, RT is only a local treatment and cannot reduce the risk of distant metastasis. Therefore, the failure to treat other organs will also affect OS and DFS.

\section{Populations Likely to Benefit From PORT}

To understand which patients benefit from PORT, researchers usually use multivariate Cox regression analysis to elucidate all relevant prognostic factors., ${ }^{7,22,32-35}$ However, these prognostic factors are often combined with other independent factors, and surgeons and radiologists may be confused when several prognostic factors coexist. To avoid the interaction between these factors and combine prognostic factors with risk of recurrence, we initially included all relevant risk factors reported $^{7,22,32-35}$ in the RPA model for risk stratification analysis. We then analyzed the role of PORT by 
hierarchical analysis, which is different from the traditional statistical analysis methods used to date.,16,19,27 RPA is one of the most successful models for stratifying patients into subgroups with homogeneous survival performance and has been performed to create prognostic algorithms for numerous malignancies. ${ }^{36,37}$

As a result, these objective risk factors (surgical margins, $\mathrm{T}$ stage, primary tumor location, and bone invasion) were confirmed to influence LRFS significantly in certain subgroups in the RPA model. The RPA model then categorized the patients with ACC into different recurrence-risk groups according to the aforementioned factors. In further stratified analysis, we found that the benefit of PORT is more obvious in intermediate- and high-risk patients. Although the difference was nonsignificant in low-risk patients, they might also benefit from PORT. Larger cases and longer follow-up time are required for further study on the benefit of PORT for low-risk patients. As a result, surgeons and radiologists can screen out patients with ACC who will benefit most from PORT while providing them with sound advice on adjuvant treatment. Furthermore, some low-risk patients can avoid overtreatment and adverse effects of RT, and treatment costs will be reduced overall.

Our study had several limitations. First, this was a retrospective study and lacks the highest clinical evidence. Second, this was an 18-year study, and treatment methods and technologies are constantly changing. Moreover, the study lacked details on radiation toxicity. Therefore, prospective studies and improved follow-up strategies are necessary for further study.

\section{Conclusions}

PORT is an important factor in improving the local control of patients with ACC but did not improve DMFS, DFS, or OS. Furthermore, we found that the benefit of PORT is more obvious in intermediate- and high-risk patients, but whether low-risk patients could benefit from PORT requires further study. This can help surgeons and radiologists provide reasonable guidance on LRFS for postoperative patients.

Submitted December 23, 2019; accepted for publication May 19, 2020.

Author contributions: Study concept and design: Y. Chen, Sun, Zhou. Visualization: Yan, Li, Huang, F.P. Chen. Funding acquisition: Sun. Data acquisition: Y. Chen, Zheng, Yan, Huang, Li. Data analysis and interpretation: Y. Chen, F.P. Chen. Software: Y. Chen. Supervision: Sun. Project administration: Sun. Manuscript preparation: Zhou, Y. Chen. Critical revision: Y. Chen, Zheng, Huang, Zhou.

Disclosures: The authors have disclosed that they have not received any financial consideration from any person or organization to support the preparation, analysis, results, or discussion of this article.

Funding: This work was supported by funding from the Health \& Medical Collaborative Innovation Project of Guangzhou City, China (number 201604020003, 201803040003); the Special Support Program of Sun Yat-sen University Cancer Center (16zxtzlc06); the Natural Science Foundation of Guangdong Province (number 2017A030312003); the Innovation Team Development Plan of the Ministry of Education (number IRT_17R110); and the National Key R\&D Program of China (2016YFC0902000)

Correspondence: Guan-Qun Zhou, MD, Department of Radiation Oncology, Sun Yat-sen University Cancer Center, State Key Laboratory of Oncology in South China, Collaborative Innovation Center of Cancer Medicine,

Guangdong Key Laboratory of Nasopharyngeal Carcinoma Diagnosis and Therapy, 651 Dongfeng Road East, Guangzhou 510060, People's Republic of China. Email: zhougq@sysucc.org.cn; and

Ying Sun, MD, Department of Radiation Oncology, Sun Yat-sen University Cancer Center; State Key Laboratory of Oncology in South China; Collaborative Innovation Center of Cancer Medicine; Guangdong Key Laboratory of Nasopharyngeal Carcinoma Diagnosis and Therapy, 651 Dongfeng Road East, Guangzhou 510060, People's Republic of China. Email: sunying@sysucc.org.cn

\section{References}

1. Thompson L. World Health Organization classification of tumours: pathology and genetics of head and neck tumours. Ear Nose Throat J 2006; 85:74.

2. Anderson JN Jr, Beenken SW, Crowe R, et al. Prognostic factors in minor salivary gland cancer. Head Neck 1995;17:480-486.

3. Meyers M, Granger B, Herman P, et al. Head and neck adenoid cystic carcinoma: a prospective multicenter REFCOR study of 95 cases. Eur Ann Otorhinolaryngol Head Neck Dis 2016;133:13-17.

4. Hsu HC, Huang EY, Wang CJ. Postoperative adjuvant radiotherapy for adenoid cystic carcinoma of the head and neck: treatment results and prognostic factors. Chang Gung Med J 2003;26:646-653.

5. Iseli TA, Karnell LH, Graham SM, et al. Role of radiotherapy in adenoid cystic carcinoma of the head and neck. J Laryngol Otol 2009;123: 1137-1144.

6. David GP, Sharon S, David A, et al. NCCN Clinical Practice Guidelines in Oncology: Head and Neck Cancers. Version 1.2020. Accessed February 12, 2020. To view the most recent version, visit NCCN.org

7. Ali S, Palmer FL, Katabi N, et al. Long-term local control rates of patients with adenoid cystic carcinoma of the head and neck managed by surgery and postoperative radiation. Laryngoscope 2017;127:2265-2269.

8. Ali S, Yeo JC, Magos T, et al. Clinical outcomes of adenoid cystic carcinoma of the head and neck: a single institution 20-year experience. J Laryngol Otol 2016;130:680-685.

9. Coca-Pelaz A, Rodrigo JP, Bradley PJ, et al. Adenoid cystic carcinoma of the head and neck-an update. Oral Oncol 2015;51:652-661.

10. Xi M, Hallemeier $\mathrm{CL}$, Merrell KW, et al. Recurrence risk stratification after preoperative chemoradiation of esophageal adenocarcinoma. Ann Surg 2018;268:289-295.

11. Suh YJ, Kwon H, Kim SJ, et al. Factors affecting the locoregional recurrence of conventional papillary thyroid carcinoma after surgery: a retrospective analysis of 3381 patients. Ann Surg Oncol 2015;22: 3543-3549

12. Fridman $\mathrm{E}, \mathrm{Na}$ 'ara $\mathrm{S}$, Agarwal J, et al. The role of adjuvant treatment in early-stage oral cavity squamous cell carcinoma: an international collaborative study. Cancer 2018;124:2948-2955.

13. Sun X, Su S, Chen C, et al. Long-term outcomes of intensity-modulated radiotherapy for 868 patients with nasopharyngeal carcinoma: an analysis of survival and treatment toxicities. Radiother Oncol 2014;110:398-403.

14. Jones AS, Hamilton JW, Rowley H, et al. Adenoid cystic carcinoma of the head and neck. Clin Otolaryngol Allied Sci 1997;22:434-443.

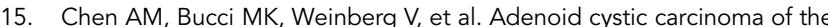
head and neck treated by surgery with or without postoperative radiation therapy: prognostic features of recurrence. Int J Radiat Oncol Biol Phys 2006;66:152-159.

16. Ouyang DQ, Liang LZ, Zheng GS, et al. Risk factors and prognosis for salivary gland adenoid cystic carcinoma in southern China: a 25-year retrospective study. Medicine (Baltimore) 2017;96:e5964.

17. Nagliati M, Bolner A, Vanoni $\mathrm{V}$, et al. Surgery and radiotherapy in the treatment of malignant parotid tumors: a retrospective multicenter study. Tumori 2009:95:442-448. 
18. Ko JJ, Siever JE, Hao D, et al. Adenoid cystic carcinoma of head and neck: clinical predictors of outcome from a Canadian centre. Curr Oncol 2016; 23:26-33.

19. van Weert S, Bloemena E, van der Waal I, et al. Adenoid cystic carcinoma of the head and neck: a single-center analysis of 105 consecutive cases over a 30-year period. Oral Oncol 2013;49:824-829.

20. Bjørndal K, Krogdahl A, Therkildsen MH, et al. Salivary adenoid cystic carcinoma in Denmark 1990-2005: outcome and independent prognostic factors including the benefit of radiotherapy. Results of the Danish Head and Neck Cancer Group (DAHANCA). Oral Oncol 2015;51:1138-1142.

21. Zhang $\mathrm{CY}, \mathrm{Xia} \mathrm{RH}, \mathrm{Han} \mathrm{J}$, et al. Adenoid cystic carcinoma of the head and neck: clinicopathologic analysis of 218 cases in a Chinese population. Oral Surg Oral Med Oral Pathol Oral Radiol 2013;115:368-375.

22. Jang S, Patel PN, Kimple RJ, et al. Clinical outcomes and prognostic factors of adenoid cystic carcinoma of the head and neck. Anticancer Res 2017:37:3045-3052.

23. Sulaiman NS, Demizu Y, Koto M, et al. Multicenter study of carbon-ion radiation therapy for adenoid cystic carcinoma of the head and neck: subanalysis of the Japan Carbon-lon Radiation Oncology Study Group (J-CROS) study (1402 HN). Int J Radiat Oncol Biol Phys 2018;100:639-646.

24. Balamucki CJ, Amdur RJ, Werning JW, et al. Adenoid cystic carcinoma of the head and neck. Am J Otolaryngol 2012;33:510-518.

25. Derer A, Frey B, Fietkau R, et al. Immune-modulating properties of ionizing radiation: rationale for the treatment of cancer by combination radiotherapy and immune checkpoint inhibitors. Cancer Immunol Immunother 2016:65:779-786.

26. Her S, Jaffray DA, Allen C. Gold nanoparticles for applications in cancer radiotherapy: mechanisms and recent advancements. Adv Drug Deliv Rev 2017;109:84-101.

27. Je HU, Song SY, Kim DK, et al. A 10-year clinical outcome of radiotherapy as an adjuvant or definitive treatment for primary tracheal adenoid cystic carcinoma. Radiat Oncol 2017;12:196.
28. Gomez DR, Hoppe BS, Wolden SL, et al. Outcomes and prognostic variables in adenoid cystic carcinoma of the head and neck: a recent experience. Int J Radiat Oncol Biol Phys 2008;70:1365-1372.

29. Cordesmeyer R, Schliephake H, Kauffmann P, et al. Clinical prognostic factors of salivary adenoid cystic carcinoma: a single-center analysis of 61 patients. J Craniomaxillofac Surg 2017;45:1784-1787.

30. Lee A, Givi B, Osborn VW, et al. Patterns of care and survival of adjuvant radiation for major salivary adenoid cystic carcinoma. Laryngoscope 2017 127:2057-2062.

31. Gurney TA, Eisele DW, Weinberg V, et al. Adenoid cystic carcinoma of the major salivary glands treated with surgery and radiation. Laryngoscope 2005; $115: 1278-1282$

32. Dantas AN, Morais EF, Macedo RA, et al. Clinicopathological characteristics and perineural invasion in adenoid cystic carcinoma: a systematic review. Rev Bras Otorrinolaringol (Engl Ed) 2015;81:329-335.

33. Ganly I, Amit M, Kou L, et al. Nomograms for predicting survival and recurrence in patients with adenoid cystic carcinoma. An international collaborative study. Eur J Cancer 2015;51:2768-2776.

34. Mannelli G, Cecconi L, Fasolati M, et al. Parotid adenoid cystic carcinoma: retrospective single institute analysis. Am J Otolaryngol 2017;38: 394-400.

35. Terhaard $\mathrm{CH}$, Lubsen $\mathrm{H}$, Rasch $\mathrm{CR}$, et al. The role of radiotherapy in the treatment of malignant salivary gland tumors. Int J Radiat Oncol Biol Phys 2005;61:103-111.

36. Huang $\mathrm{SH}, \mathrm{Xu}$ W, Waldron J, et al. Refining American Joint Committee on Cancer/Union for International Cancer Control TNM stage and prognostic groups for human papillomavirus-related oropharyngeal carcinomas. $\mathrm{J}$ Clin Oncol 2015;33:836-845.

37. Carson KA, Grossman SA, Fisher JD, et al. Prognostic factors for survival in adult patients with recurrent glioma enrolled onto the new approaches to brain tumor therapy CNS consortium phase I and II clinical trials. J Clin Oncol 2007;25:2601-2606.

\section{See JNCCN.org for supplemental online content.}


Supplemental online content for:

\section{Role of Postoperative Radiotherapy in Nonmetastatic Head and Neck Adenoid Cystic Carcinoma}

Yue Chen, MD; Zi-Qi Zheng, MD; Fo-Ping Chen, MD; Jian-Ye Yan, BA; Xiao-Dan Huang, MD; Feng Li, MD;

Ying Sun, MD; and Guan-Qun Zhou, MD

J Natl Compr Canc Netw 2020;18(11):1476-1484

eFigure 1: Representative Clinical Target Volume of a 33-Year-Old Woman With Adenoid Cystic Carcinoma of Parotid Gland Without Nerve Invasion

eFigure 2: Representative Clinical Target Volume of a 52-Year-Old Woman With Adenoid Cystic Carcinoma of Submandibular Gland With Nerve Invasion

eFigure 3: Kaplan-Meier Estimates of Distant Metastasis-Free Survival in Different Prognostic Groups

eTable 1: Primary Tumor Locations Among Study Cohort 

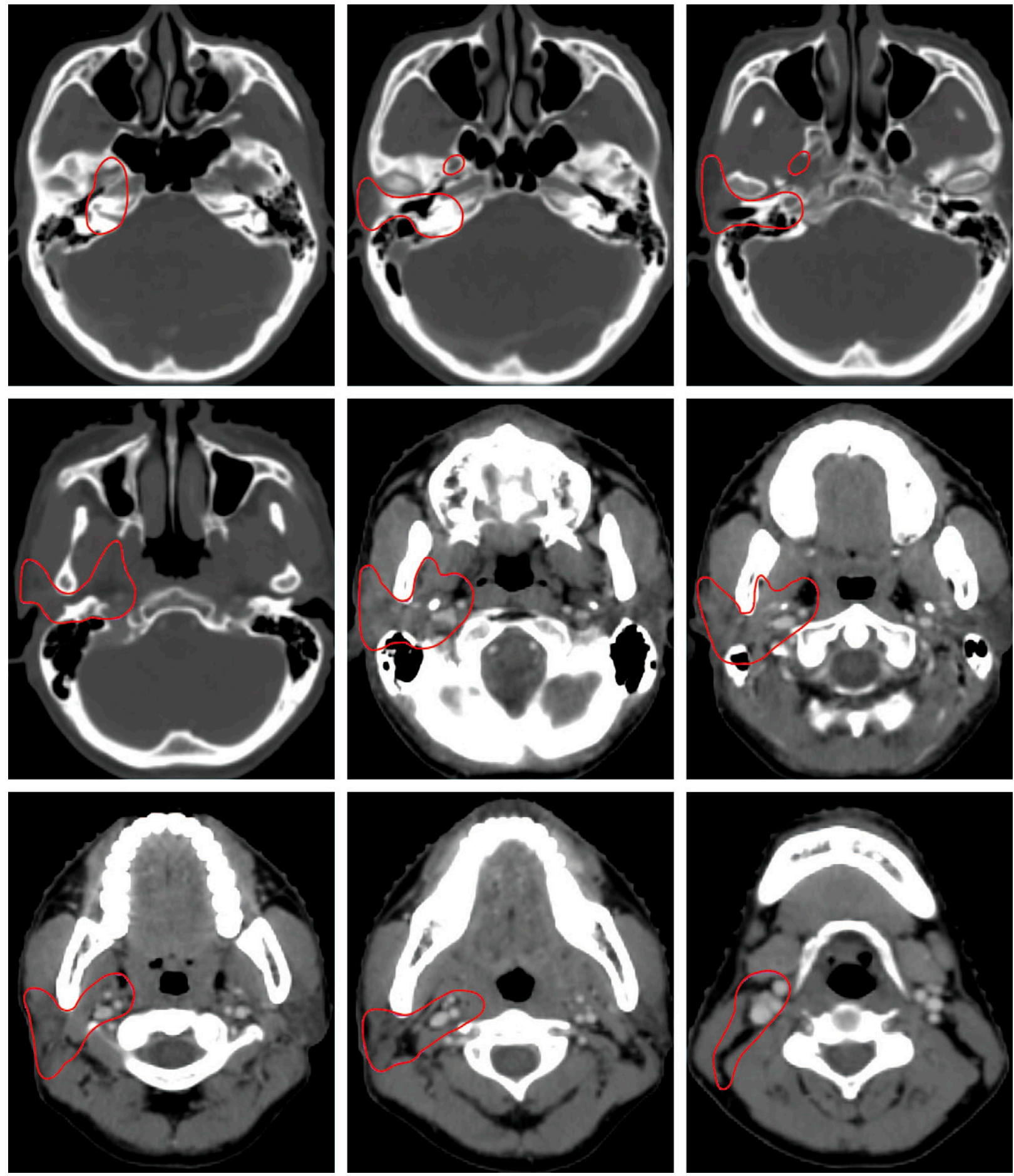

eFigure 1. Representative clinical target volume of a 33-year-old woman with adenoid cystic carcinoma of parotid gland without nerve invasion (red line represents the area of the target). 

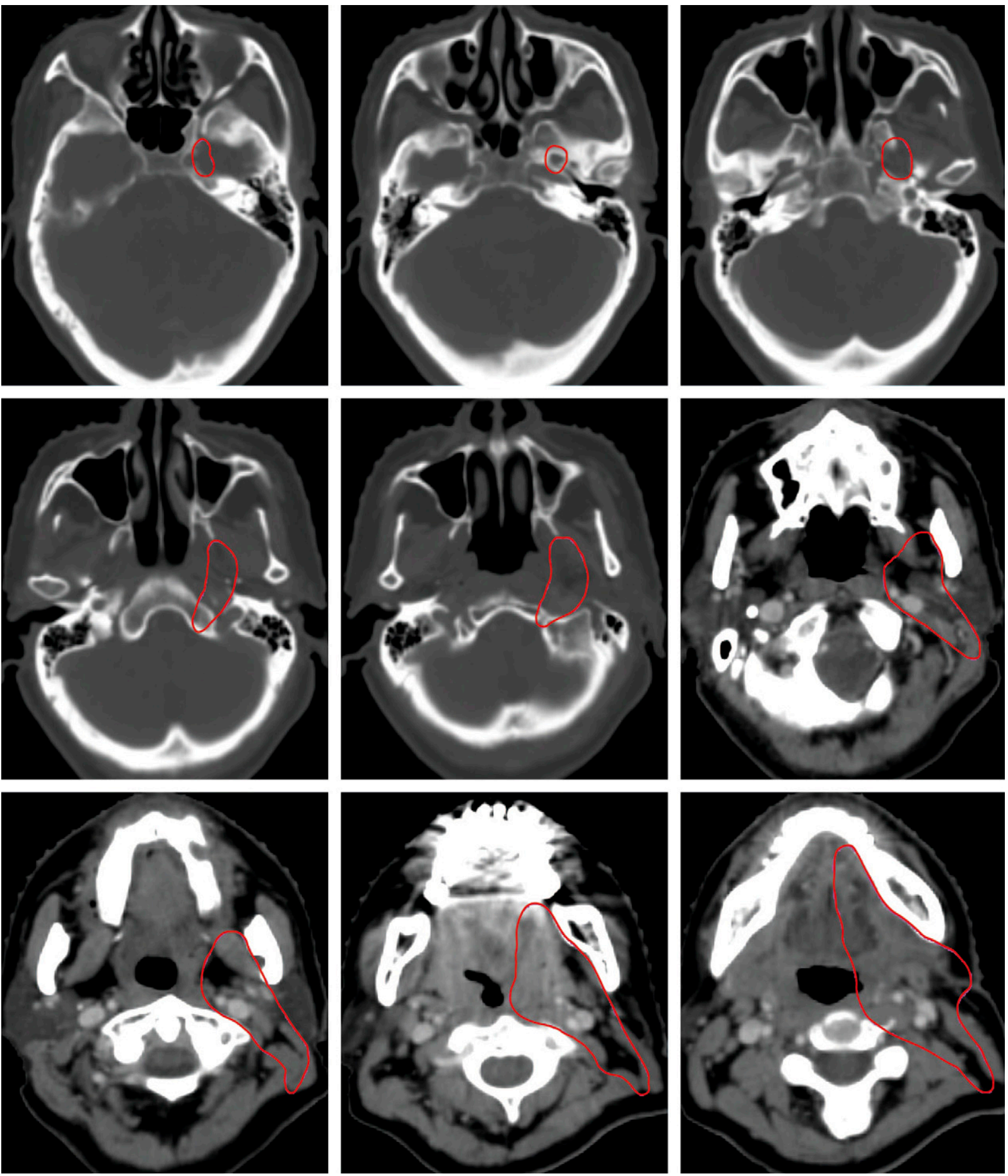

eFigure 2. Representative clinical target volume of a 52-year-old woman with adenoid cystic carcinoma of submandibular gland with nerve invasion (red line represents the area of the target). 


\section{A}

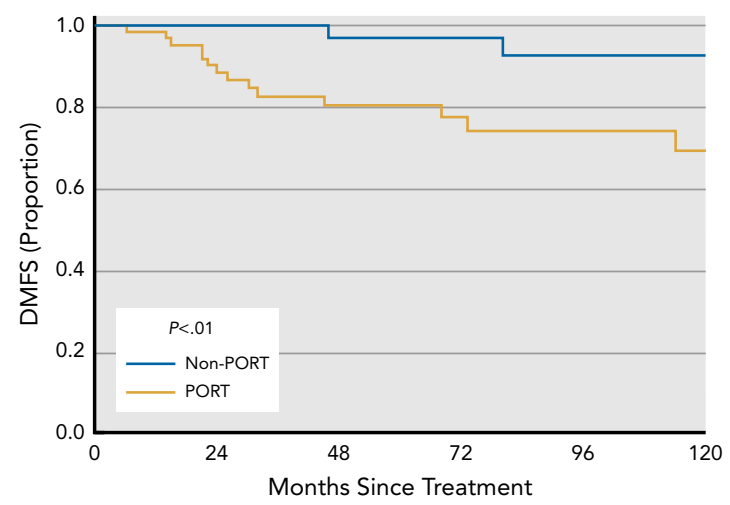

Number at risk

$\begin{array}{rllllll}\text { non-PORT } & 45 & 38 & 34 & 26 & 19 & 13 \\ \text { PORT } & 67 & 51 & 35 & 25 & 20 & 15\end{array}$

B

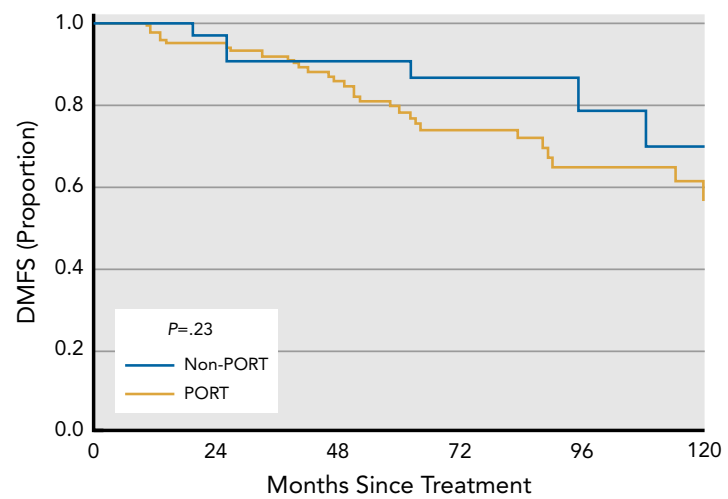

Number at risk

\begin{tabular}{|c|c|c|c|}
\hline non-PORT 38 & 32 & 23 & 17 \\
\hline PORT 129 & 99 & 74 & 45 \\
\hline
\end{tabular}

C

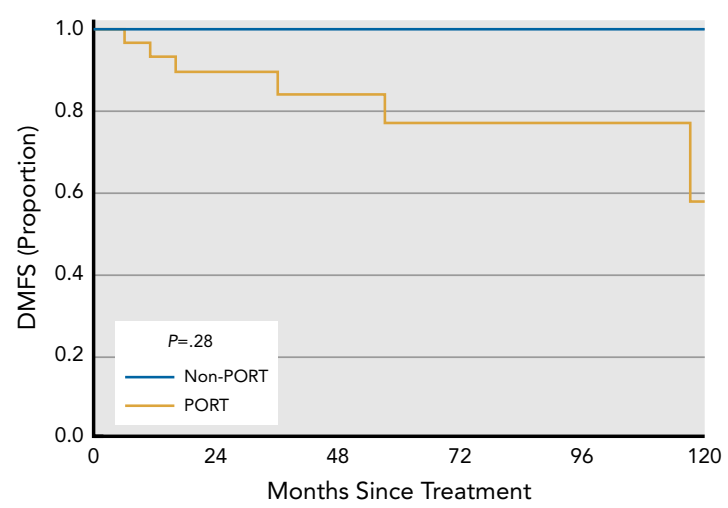

Number at risk

$\begin{array}{rrrrrrr}\text { non-PORT } & 9 & 7 & 3 & 2 & 2 & 2 \\ \text { PORT } & 31 & 23 & 15 & 9 & 8 & 4\end{array}$

eFigure 3. Kaplan-Meier estimates of the role of PORT in DMFS in the (A) low-risk, (B) intermediate-risk, and (C) high-risk groups, derived by recursive partitioning analysis.

Abbreviations: DMFS, distant metastasis-free survival; PORT, postoperative radiotherapy. 
eTable 1. Primary Tumor Locations Among Study Cohort

Primary Tumor Location

n (\%)

$\begin{array}{ll}\text { Total, N } & 319\end{array}$

Major salivary gland

Parotid 52 (16.3)

Submandibular 33 (10.3)

Sublingual 12 (3.8)

Minor salivary gland

Hard/Soft palate $68(21.3)$

Floor of mouth 35 (11.0)

Buccal 33 (10.3)

Mobile tongue 15 (4.7)

Lip 15 (4.7)

Retromolar 12 (3.8)

Maxilla 8 (2.5)

Mandible $3(0.9)$

Other

Eye socket $\quad 5(1.6)$

Maxillary sinus $\quad 5(1.6)$

Base of tongue 5 (1.6)

Nasal cavity $\quad 4$ (1.3)

Oropharynx $4(1.3)$

Nasopharynx $3(0.9)$

Facial skin $2(0.6)$

Ethmoidal sinus $1(0.3)$

Paranasal sinus $1(0.3)$

Hypopharynx $1(0.3)$

Larynx $1(0.3)$

Parapharyngeal space $\quad 1(0.3)$ 